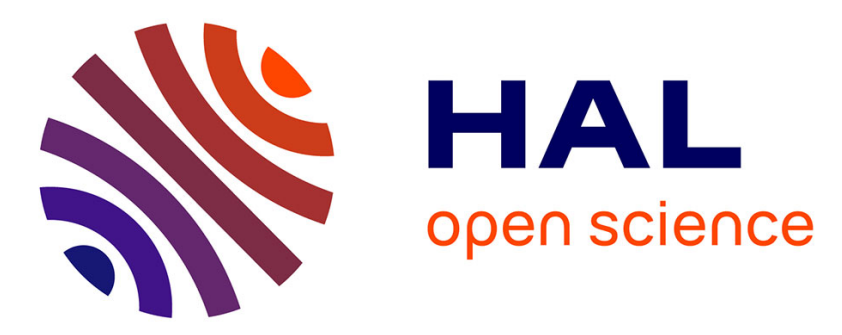

\title{
Réflectométrie appliquée aux interfaces diffuses : possibilités et limites de la technique
}

\author{
P. Schaaf, Ph. Dejardin, Anne Schmitt
}

\section{To cite this version:}

P. Schaaf, Ph. Dejardin, Anne Schmitt. Réflectométrie appliquée aux interfaces diffuses: possibilités et limites de la technique. Revue de Physique Appliquée, 1985, 20 (9), pp.631-640. 10.1051/rphysap:01985002009063100 . jpa-00245378

\section{HAL Id: jpa-00245378 https://hal.science/jpa-00245378}

Submitted on 1 Jan 1985

HAL is a multi-disciplinary open access archive for the deposit and dissemination of scientific research documents, whether they are published or not. The documents may come from teaching and research institutions in France or abroad, or from public or private research centers.
L'archive ouverte pluridisciplinaire HAL, est destinée au dépôt et à la diffusion de documents scientifiques de niveau recherche, publiés ou non, émanant des établissements d'enseignement et de recherche français ou étrangers, des laboratoires publics ou privés. 


\title{
REVUE DE PHYSIQUE APPLIQUÉE
}

\section{Réflectométrie appliquée aux interfaces diffuses : possibilités et limites de la technique}

\author{
P. Schaaf, Ph. Dejardin et A. Schmitt \\ Institut Charles Sadron (CRM-EAHP), CNRS-ULP Strasbourg, \\ 6, rue Boussingault, 67083 Strasbourg Cedex, France
}

(Reçu le 19 mars 1985, révisé le 5 juin, accepté le 10 juin 1985)

\begin{abstract}
Résumé. - La réflectivité d'interfaces diffuses correspondant à un profil d'indice de réfraction sigmoïdal est analysée, dans le but d'explorer les possibilités et les limites de la technique. Différentes méthodes de calcul des coefficients de réflectivité sont d'abord comparées, celle proposée par Abelès étant de validité la plus générale. Pour des ondes « $s$ » et dans le cas de profils interfaciaux centrosymétriques, le coefficient de réflectivité est presque directement proportionnel au coefficient de réflectivité de Fresnel et à la transformée de Fourier de la dérivée du profil d'indice; cette approximation est très bonne dans la plupart des situations expérimentales rencontrées. Par contre, ce résultat n'est plus valable pour des profils dissymétriques. Nous montrons que l'étude conjointe de la réflectivité des ondes « $s$ » et " $p$ » en fonction de l'angle d'incidence permet, en principe, la détermination d'une épaisseur caractéristique du profil et de son paramètre de dissymétrie.
\end{abstract}

\begin{abstract}
We analyse the reflectivity of diffuse interfaces corresponding to a sigmoidal refraction index profile, in order to explore the possibilities and the limits of the technique. We first compare various methods of calculation of the reflectivity coefficients, and show that the general method developed by Abelès is the most reliable. For centrosymmetric profiles, the reflectivity coefficient of « $s$ » waves is almost directly proportional to the Fresnel reflectivity coefficient and to the Fourier transform of the derivative of the refractive index profile, under most experimental conditions encountered. Yet, this result is no more valid for non-symmetric profiles. Finally, we show that a combined study of « $s$ " and « $p$ " reflectivities allows, in principle, the determination of two characteristic parameters of the interfacial profile : thickness and dissymmetry coefficient.
\end{abstract}

\section{Introduction.}

Dans l'étude de la structure des interfaces, les techniques optiques occupent une place de choix [1-4]. Complémentaires des techniques hydrodynamiques en ce qui concerne les polymères adsorbés à une interface solide/liquide [5], elles permettent aussi la caractérisation structurale des interfaces de type liquide/liquide [6] et liquide/vapeur [7, 8].

L'ellipsométrie a connu un essor particulier durant la dernière décennie [9]. Ce succès est justifié par la sensibilité de cette technique qui permet la mesure précise d'épaisseurs très faibles allant jusqu'au $\mathrm{nm}$. A l'opposé, le développement de l'étude des phéno- mènes critiques a conduit plusieurs auteurs à étudier le voisinage du point critique de démixtion de deux phases fluides : l'épaisseur de l'interface est alors conséquente, et la différence relative d'indice de réfraction entre les deux milieux faible. En ce cas, la méthode de réflectométrie sous incidence normale, développée par Webb et ses collaborateurs [6] est $a$ priori bien adaptée. Le calcul du coefficient de réflectivité (rapport $r$ des amplitudes des champs électriques relatifs aux ondes réfléchie et incidente) s'effectue à partir d'une formule proposée par Webb [6], bien que son domaine de validité n'ait pas été clairement établi.

L'objectif du présent article est d'explorer plus 
largement les possibilités de la technique de réflectométrie, dans la perspective de son application potentielle à l'étude d'interfaces contenant des polymères. En nous limitant pour l'instant aux interfaces intervenant dans les problèmes de démixtion, il est clair que le voisinage du point critique ne constitue pas le seul domaine d'étude intéressant. Lorsqu'on s'écarte plus fortement de la température critique $T_{\mathrm{c}}$, la structure des interfaces mérite d'être étudiée (épaisseur, mais aussi éventuelle dissymétrie) dans des conditions où la variation relative d'indice d'une phase homogène à l'autre peut devenir conséquente. Par ailleurs, du point de vue expérimental, il est possible d'étudier la réflectivité en fonction de l'angle d'incidence, et de mesurer avec précision des coefficients de réflectivité très faibles. Aussi importe-t-il de tirer parti, au niveau de calculs théoriques, de ces possibilités expérimentales.

Dans l'analyse qui suit nous comparerons, dans un premier chapitre, différentes méthodes de calcul des coefficients de réflectivité, en tentant de dégager leurs domaines de validité respectifs. Dans une seconde partie, nous présenterons quelques résultats significatifs de nos calculs. Ils devraient conduire, pensons-nous, à une expérimentation plus éclairée et à une analyse plus approfondie des résultats expérimentaux obtenus.

\section{Calcul de la réflectivité d'une interface diffuse.}

Il existe de nombreux travaux sur la propagation des ondes acoustiques ou électromagnétiques dans l'atmosphère, le milieu marin ou sous terre [10]; il est alors légitime d'utiliser des approximations fondées sur le fait que la longueur d'onde $\lambda$ est petite devant les distances sur lesquelles les propriétés du milieu varient de manière significative. Pour une onde électromagnétique dans un milieu transparent, cette condition se traduit en tout point $\mathbf{r}$ par :

$$
\lambda(\mathbf{r}) \ll|\nabla[\ln n(\mathbf{r})]|^{-1}
$$

$n(r)$ étant l'indice du milieu en ce point. Pour nombre d'interfaces de type fluide/fluide ou fluide/solide, la situation physique est intermédiaire entre le cas précédent (Eq. (1)) et celui considéré par Fresnel, à savoir l'interface discrète où l'indice varie brusquement de la valeur $n_{1}$ (milieu incident) à la valeur $n_{2}$ (milieu réfracté). Dans le cas d'interfaces planes on définit alors le profil d'indice $n(z), z$ désignant l'abscisse sur un axe $z^{\prime} z$ orthogonal à l'interface, le système étant supposé homogène dans tout plan orthogonal à $z^{\prime} z$. Les valeurs des coefficients de réflectivité en amplitude $r_{\mathrm{s}}$ et $r_{\mathrm{p}}$, relatifs aux ondes $" \mathrm{~s}$ " et " $\mathrm{p}$ " (vecteurs champ électrique respectivement orthogonal et parallèle au plan d'incidence) sont alors inférieures (sauf près de l'angle de Brewster, pour des ondes " $p$ ") à celles calculées selon les formules de Fresnel. L'écart est d'autant plus important que le profil est plus étendu. De ce fait, c'est la largeur du profil qui constitue la première grandeur à mesurer, soit par ellipsométrie, soit par réflectométrie.

\subsection{ProfiLS D'INDICE.}

\subsubsection{Profil linéaire. - Il est caractérisé par}

$$
\begin{aligned}
& n(z)=n_{2}, z \leqslant-b / 2 ; n(z)=n_{1}, z \geqslant b / 2 \\
& n(z)=\frac{n_{1}+n_{2}}{2}-\left(\frac{n_{2}-n_{1}}{b}\right) z,-b / 2 \leqslant z \leqslant b / 2 .
\end{aligned}
$$

Un tel profil d'indice est physiquement peu réaliste, quoiqu'il puisse être approché dans des problèmes de diffusion stationnaire entre deux milieux à conditions aux limites imposées (température, concentration), ou lors de phénomènes de polarisation interfaciale $[11,12]$. Nous l'avons toutefois choisi par commodité, car il présente des bornes d'intégration bien définies. Remarquons qu'il existe, pour un tel profil de potentiel, une solution non triviale de l'équation de Schrödinger [13].

2.1.2 Profil sigmoïdal. - Les équations le définissant sont :

$$
\begin{array}{ll}
n(z)=\left[n_{1}^{2}+\left(n_{0}^{2}-n_{1}^{2}\right) \exp \left(-z / b_{1}\right)\right]^{1 / 2}, & z \geqslant 0 \\
n(z)=\left[n_{2}^{2}-\left(n_{2}^{2}-n_{0}^{2}\right) \exp \left(+z / b_{2}\right)\right]^{1 / 2}, & z \leqslant 0
\end{array}
$$

$n_{0}$ est l'indice en $z=0$, où l'on impose de plus la condition de continuité des dérivées premières :

$$
\left(n_{0}^{2}-n_{1}^{2}\right) b_{2}=\left(n_{2}^{2}-n_{0}^{2}\right) b_{1} .
$$

Les valeurs asymptotiques des indices $\left(n_{1}\right.$ et $\left.n_{2}\right)$ et celles des longueurs caractéristiques $\left(b_{1}\right.$ et $\left.b_{2}\right)$ étant données, cette équation détermine $n_{0}$.

Pareil profil est aussi défini par un paramètre de dissymétrie $X$ tel que :

$$
X=\frac{b_{1}-b_{2}}{b_{1}+b_{2}}, \quad-1<X<+1 .
$$

Lorsque $X=0$, c'est le profil de la fonction permittivité $\varepsilon(z)=[n(z)]^{2}$ qui est symétrique; mais il en est pratiquement de même pour la fonction $n(z)$ à condition que

$$
\frac{n_{2}-n_{1}}{n_{2}+n_{1}}=\frac{\Delta n}{2 \bar{n}}=r_{F} \text { reste assez faible }
$$

$r_{\mathrm{F}}$ étant la réflectivité de Fresnel sous incidence normale.

Deux raisons justifient le choix d'un tel profil d'indice. On peut montrer dans les problèmes de séparation de phase faisant intervenir des systèmes polymères que la composition de l'interface tend exponentiellement vers celles de l'un et l'autre milieu, avec des longueurs caractéristiques qui ne sont pas a priori les mêmes, sauf au voisinage du point critique [14]. Par ailleurs, nous avons pu calculer l'expression 
analytique du coefficient de réflectivité $r_{\mathrm{s}}$, dans le cas d'un tel profil [15]. Ce résultat constituc ainsi une bonne référence pour les calculs.

2.1.3 Epaisseurs. - Nous définissons l'épaisseur d'une interface à partir des moments de la fonction de distribution $\mathrm{d} n(z) / \mathrm{d} z$, qui tend asymptotiquement vers zéro dans les milieux extrêmes. Si l'on note $\left\langle z^{m}\right\rangle$ le moment d'ordre $m$ de la distance à l'interface [16],

$$
\left\langle z^{m}\right\rangle=\int_{-\infty}^{+\infty} z^{m} \frac{\mathrm{d} n}{\mathrm{~d} z} \cdot \mathrm{d} z / \int_{-\infty}^{+\infty} \frac{\mathrm{d} n}{\mathrm{~d} z} \cdot \mathrm{d} z
$$

l'épaisseur $L$ est définie comme étant la racine carrée de la dispersion quadratique moyenne des distances :

$$
L=\left[\left\langle z^{2}\right\rangle-\langle z\rangle^{2}\right]^{1 / 2} .
$$

Pour le profil linéaire (Eq. (2)) on obtient

$$
L=b / 2 \sqrt{3}
$$

alors que pour le profil sigmoïdal il n'existe pas, à notre connaissance, de relation analytique simple. Aussi est-il nécessaire de calculer $L$ par ordinateur. Précisons incidemment que pour un " profil en tanh »

$$
n(z)=n_{0}+\frac{\Delta n}{2} \tanh \left(\frac{x}{\xi \sqrt{2}}\right)
$$

tel qu'on le rencontre au voisinage du point critique de démixtion, dans les problèmes de mélanges de polymères [17], l'épaisseur est liée à $\xi$ par :

$$
L=\frac{\Pi \xi}{\sqrt{6}} .
$$

2.2 MÉTHODES D'ABELÈs POUR LE CALCUL DES COEFFICIENTS DE RÉFleCtIVITÉ $r_{i}(i=\mathrm{s}, \mathrm{p})$. - Prenons le plan $Y O Z$ comme plan d'incidence (Fig. 1). Abelès montre [18] que, pour une onde sinusoïdale de longueur d'onde $\lambda$ et de pulsation $\omega$, polarisée perpendiculairement au plan d'incidence, les composantes $E_{x}$ et $H_{y}$ des champs électrique $\mathbf{E}$ et magnétique $\mathbf{H}$, dans les plans homogènes d'indices $n_{1}$ et $n_{2}$, sont reliées par une matrice $(M)$ fonction du profil d'indice $n(z)$. Les composantes $E_{x}$ et $H_{y}$ s'écrivent :

$$
\begin{aligned}
& E_{x}(y, z, t)=U(z) \exp [i(\omega t-S y)] \\
& H_{y}(y, z, t)=V(z) \exp [i(\omega t-S y)]
\end{aligned}
$$

avec

$$
S=k_{1} \sin \theta_{1}=k_{2} \sin \theta_{2} ; \quad k_{i}=\frac{2 \pi n_{i}}{\lambda}(i=1,2)
$$

$\theta_{1}$ et $\theta_{2}$ étant respectivement les angles d'incidence et de réfraction. En se fixant une valeur de $U_{2}(z)$ caractéristique de l'onde réfractée, loin de l'interface $(z \ll-L)$, c'est-à-dire en milieu homogène, on déter-

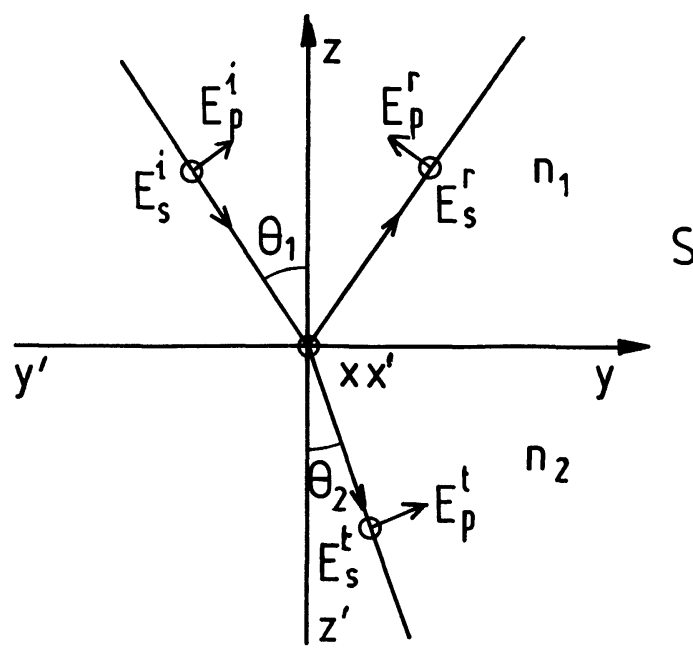

Fig. 1. - Représentation schématique des composantes $s$ et $p$ des champs incident et réfléchi (milieu 1) et réfracté (milieu 2), le plan $X O Y$ représentant le plan de l'interface.

[Schematic representation of the $s$ and $p$ components of the electrical field in the incident, reflected (phase 1) and refracted (phase 2) waves, the interface being located in the $X O Y$ plane.]

mine les composantes du champ magnétique $\mathbf{H}_{2}$ par l'intermédiaire de la relation :

$$
\mathbf{H}_{2}=\frac{\mathbf{k}_{2} \wedge \mathbf{E}_{2}}{\mu_{0} \omega}
$$

En milieu incident, les amplitudes complexes $U_{1}(z)$ et $V_{1}(z)$ s'obtiennent, très loin de l'interface $(z \gg L)$ par le biais de la relation matricielle

$$
\left[\begin{array}{l}
U_{1} \\
V_{1}
\end{array}\right]=\left(M_{\mathrm{s}}\right)\left[\begin{array}{c}
U_{2} \\
V_{2}
\end{array}\right]
$$

D'où l'expression du coefficient de réflectivité en amplitude :

$$
r_{\mathrm{s}}=\frac{U_{1}-V_{1} \frac{\sqrt{\mu_{0} / \varepsilon_{0}}}{n_{1} \cos \theta_{1}}}{U_{1}+V_{1} \frac{\sqrt{\mu_{0} / \varepsilon_{0}}}{n_{1} \cos \theta_{1}}}
$$

Pour une onde polarisée parallèlement au plan d'incidence, les composantes des champs magnétique et électrique s'écrivent :

$$
\begin{aligned}
& H_{x}=U(z) \mathrm{e}^{i(\omega t-S y)} \\
& E_{y}=-V(z) \mathrm{e}^{i(\omega t-S y)}
\end{aligned}
$$

La même démarche que celle résumée ci-dessus conduit à l'expression du coefficient de réflectivité $r_{\mathrm{p}}$, lorsqu'on connaît la matrice de transfert $\left(M_{p}\right)$ : 


$$
r_{\mathrm{p}}=\frac{U_{1}-V_{1} \frac{n_{1}}{\cos \theta_{1}} \sqrt{\varepsilon_{0} / \mu_{0}}}{U_{1}+V_{1} \frac{n_{1}}{\cos \theta_{1}} \sqrt{\varepsilon_{0} / \mu_{0}}}
$$

La difficulté essentielle réside donc dans l'évaluation de la matrice $(M)$ caractéristique de la structure de l'interface. Deux méthodes ont été proposées par Abelès [18].

2.2.1 Méthode d'Abelès, dite des cosinus (MAC). Elle consiste à décomposer l'interface en tranches de faible épaisseur $t_{j}$, chaque tranche supposée homogène étant caractérisée par un indice de réfraction $n_{j}$ et une matrice de transfert $M_{j}$. La matrice relative à toute l'interface s'écrit alors

$$
M=\prod_{j} M_{j} .
$$

Nous rappelons en appendice I l'expression des matrices $M_{j \mathrm{~s}}$ et $M_{j \mathrm{p}}$ relatives à des ondes « $\mathrm{s}$ » et « $\mathrm{p}$ ».
Dans les calculs qui suivent, nous adoptons pour valeur de longueur d'onde dans le vide $\lambda_{0}=6328 \AA$ (laser $\mathrm{He}-\mathrm{Ne}$ ) et les indices des milieux extrêmes seront, sauf indication contraire, $n_{1}=1,4$ et $n_{2}=1,6$, indices voisins de ceux du cyclohexane et du polystyrène purs, respectivement. Avec la méthode MAC, nous avons effectué, pour des profils sigmoïdaux et des ondes de types « $\mathrm{s}$ », des calculs de coefficients de réflectivité. Les résultats ont été comparés, pour différentes épaisseurs et différents angles d'incidence, au résultat exact établi pour ce type de profil [15]. L'accord entre les deux méthodes de calcul est très bon, et deux observations sont à relever :

- Si l'on veut réduire l'écart relatif entre les deux résultats à moins que $10^{-5}$, il convient pour $L=800 \AA$, de découper le profil en tranches d'épaisseur voisine de $1 \AA$, cette limitation est imposée par le fort gradient d'indice au point d'inflexion.

- Le choix de bornes d'intégration est conditionné par la précision que l'on désire atteindre au niveau des

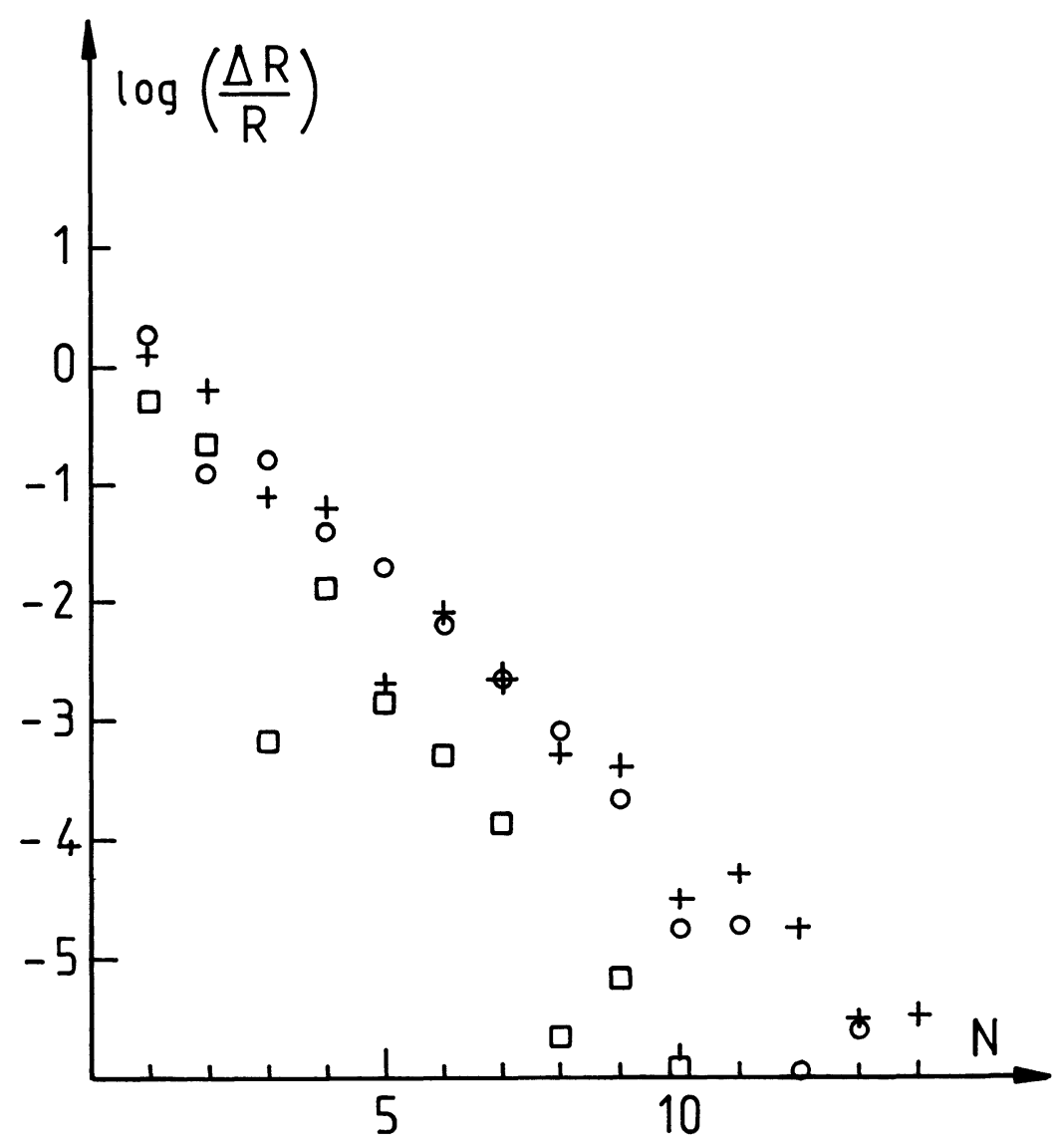

Fig. 2. - Variations de la valeur absolue de l'erreur relative sur le calcul du coefficient de réflectivité en énergie, sous incidence normale (échelle logarithmique décimale), avec le paramètre d'intégration $N$ (intégration entre $\pm N L$ ), pour $L=600 \AA$, $n=0,2$, et pour différentes valeurs du paramètre de dissymétrie $X$.

$$
+, X=-0,906 ; \text { O, } X=0,893 ; \square, X=-0,033 \text {. }
$$

[Variations of the absolute value of the relative error related to the energy reflection coefficient under normal incidence (decimal logarithmic scale) with the integration parameter $N$ (integration between $\pm N L$ ), for $L=600 \AA, n=0.2$, and for different values of the dissymmetry parameter $X$.

$$
+, X=-0.906 ; \quad \text { O, } X=0.893 ; \square, X=-0,033 .]
$$


calculs. La figure 2 illustre les variations de la valeur absolue l'erreur relative $\Delta R / R$ avec le coefficient $N$ (les bornes d'intégration étant $\pm N L$ ). On remarque que les "relaxations" asymptotiques des concentrations dans les milieux incident et réfracté apportent une contribution importante à la réflectivité. En effet, si l'on désire un résultat défini à mieux que $1 \%$, il convient de choisir $N>5$.

2.2.2 Méthode Abelès d'intégration (MAI). - Les éléments de la matrice $M$ sont calculés au moyen d'intégrales faisant intervenir la fonction $\varepsilon(z)$ [18], c'est-à-dire le profil de permittivité diélectrique. L'intérêt de la méthode réside dans le fait qu'elle utilise pour le calcul de $M$, des développements en puissances paires de la norme $k_{0}$ du vecteur d'onde dans le vide. Or, Abelès rappelle que la formule de Drude [19] relative au coefficient ellipsométrique se déduit de ce développement en se limitant à l'ordre 1 en $k_{0}^{2}$.

Pour un profil linéaire, sous incidence normale, nous avons calculé la réflectivité en fonction de l'épaisseur à différents ordres en $k_{0}^{2}$, et comparé les résultats des méthodes MAI et MAC. Le pas choisi pour les calculs sur ordinateur est toujours de $1 \AA$.

Les résultats présentés dans le tableau I font ressortir que, si l'on requiert une précision expérimentale de $1 \%$ sur le coefficient de réflectivité en intensité, l'approximation d'ordre 1 suffit jusqu'à des épaisseurs de l'ordre de $100 \AA$. Pour une interface diffuse linéaire, d'épaisseur $400 \AA$, il est par contre nécessaire, dans les mêmes conditions, de développer les calculs jusqu'à l'ordre 4, soit bien au-delà de l'approximation retenue par Drude. Le tableau I présente cependant plus de chiffres significatifs, afin de mieux situer les problèmes de convergence.

Bref, dans le cadre expérimental où nous nous sommes placés $(\Delta n=0,2)$, la méthode MAI n'est intéressante que pour les faibles épaisseurs.

De fait, il apparaît plus fécond, lorsque $\Delta n / n$ est faible, d'utiliser des méthodes s'inspirant de l'approximation de Born. C'est ce qu'ont fait Webb [6] pour la réflectométrie, et plus récemment de Gennes et Charmet [20] pour l'ellipsométrie.
2.3 Formule approchée de Webb [6]. - Pour le calcul de $r$ au voisinage d'une température critique de démixtion, Webb propose la formule suivante, dans le cas d'une incidence normale :

$$
r=\left|\frac{1}{2 \bar{n}} \int_{-\infty}^{+\infty} \frac{\mathrm{d} n}{\mathrm{~d} z} \exp (-2 i k(z) z) \mathrm{d} z\right|
$$

$\bar{n}$ est l'indice moyen des deux milieux, et $k(z)$ la norme du vecteur d'onde au point d'abscisse $z$.

Si l'on pose :

$$
\text { , } n(z)=n_{1}+\Delta n . f(z) .
$$

Il apparaît que

$$
\lim _{\Delta n \rightarrow 0}\left(\frac{r}{r_{F}}\right)=\hat{f}^{\prime}(2 k)
$$

où $\hat{f}^{\prime}(2 k)$ est la transformée de Fourier de la fonction de distribution $\mathrm{d} f / \mathrm{d} z$.

Sur les figures $3 a$ et $3 b$, nous avons représenté l'écart relatif entre le coefficient de réflectivité en énergie calculé selon la méthode de Webb et le résultat exact, pour des profils sigmoïdaux et deux valeurs différentes de l'écart $\Delta n$. L'approximation de Webb est excellente pour des profils symétriques, quelle que soit l'épaisseur, pour les différences d'indice $\Delta n$ rencontrées expérimentalement. De plus, cette approximation reste bonne quel que soit le profil (symétrique ou non symétrique) pour $\Delta n<0,2$, ainsi que l'illustre la figure 3a. Par contre, pour $\Delta n>0,2$, la formule de Webb n'est plus satisfaisante pour les profils dissymétriques (voir Fig. 3b). Certes, il s'agit là de conditions extrêmes, probablement peu rencontrées expérimentalement. Néanmoins, le phénomène d'adsorption à une interface solide/liquide ou liquide/air fait intervenir des profils qui sont bien plus dissymétriques [21].

Lorsqu'on s'écarte de l'incidence normale, les formules proposées par Webb ne sont plus applicables qu'au voisinage immédiat du point critique, l'incidence brewstérienne se situant toujours à $\theta=\pi / 2[6]$. Webb ne fournit d'ailleurs pas de démonstration de ces rela-

Tableau I. - Coefficients de réflectivité $r_{\mathrm{s}}(\times 100)$ calculés selon différentes méthodes, pour un profil d'indice linéaire.

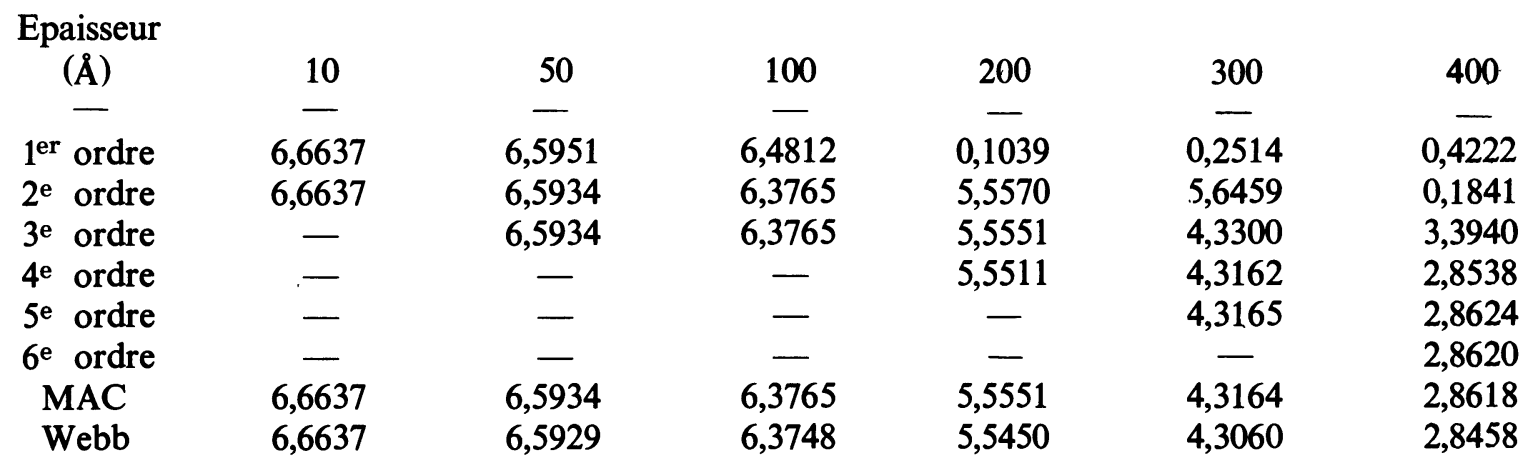




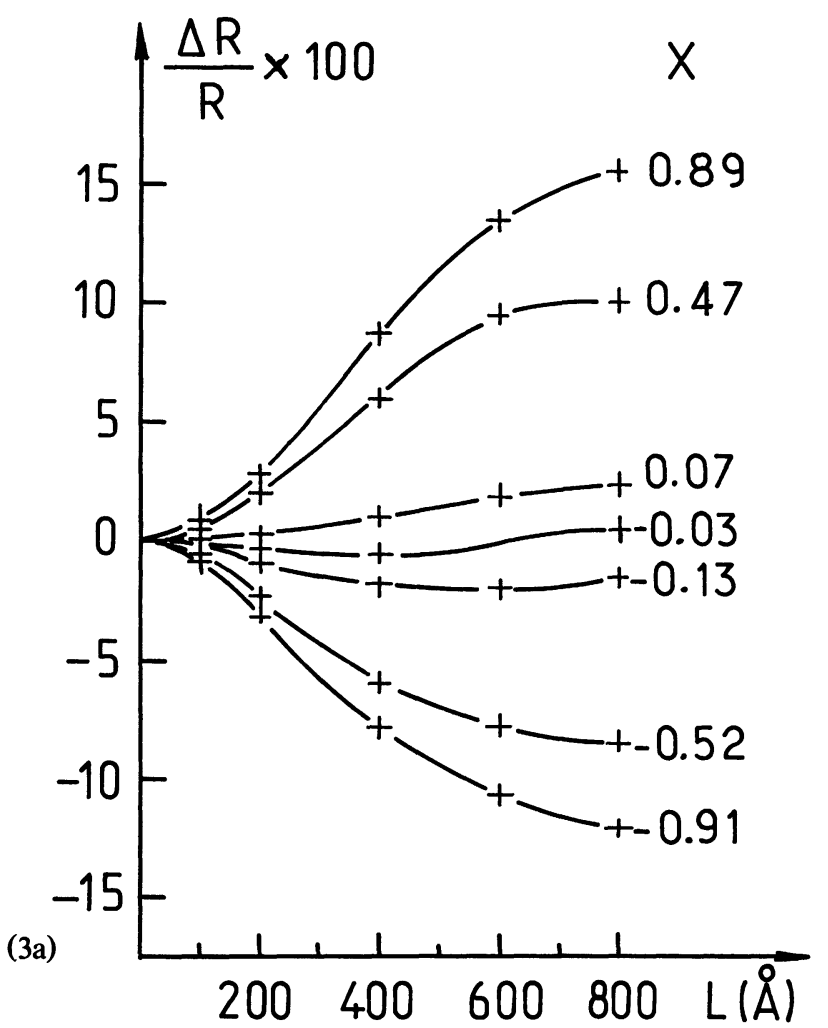

Figs. 3a et 3b. - Différences relatives (en \%) entre le coefficient de réflectivité calculé selon la méthode approchée de Webb (incidence normale) et sa valeur exacte, en fonction de l'épaisseur du profil sigmoïdal, pour différentes valeurs du paramètre de dissymétrie $X$ (cf. Eq. (5)); Fig. 3a : $\Delta n=0,2$; Fig. $3 \mathrm{~b}: \Delta n=0,02$.

tions. C'est pourquoi nous proposons en appendice II une clarification sur ce sujet.

\section{Réflectométrie d'une interface diffuse.}

L'analyse qui précède constitue une mise au point sur les méthodes de calcul, et permet d'étudier l'influence des paramètres physiques $(\Delta n)$ et géométriques $\left(L, X\right.$, angle d'incidence $\left.\theta_{\mathrm{i}}\right)$ caractérisant la réflectivité d'une interface diffuse. Envisageons successivement des ondes $s$ et $p$, pour lesquelles le coefficient de réflexion peut être calculé soit directement [15] (ondes s) soit par la méthode d'Abelès MAC (ondes p) dans le cas du profil d'indice sigmoïdal défini par l'équation (3).

3.1 ONDES DE TYPE « $\mathrm{s}$ ». - Considérons les variations du coefficient de réflectivité en énergie $R_{\mathrm{s}}$ avec l'angle d'incidence, pour différentes épaisseurs $L$ et dissymétries $X$. Elles sont représentées sur la figure 4 et conduisent aux observations suivantes :

- Pour des épaisseurs inférieures à $100 \AA$, le rapport $R_{\mathrm{s}} / R_{\mathrm{Fs}}$ (où $R_{\mathrm{Fs}}$ est la réflectivité de Fresnel en énergie) est très proche de 1 , quel que soit l'angle d'incidence. L'étude par réflectométrie des ondes $s$ n'est donc pas adaptée dans ce domaine d'épaisseurs.

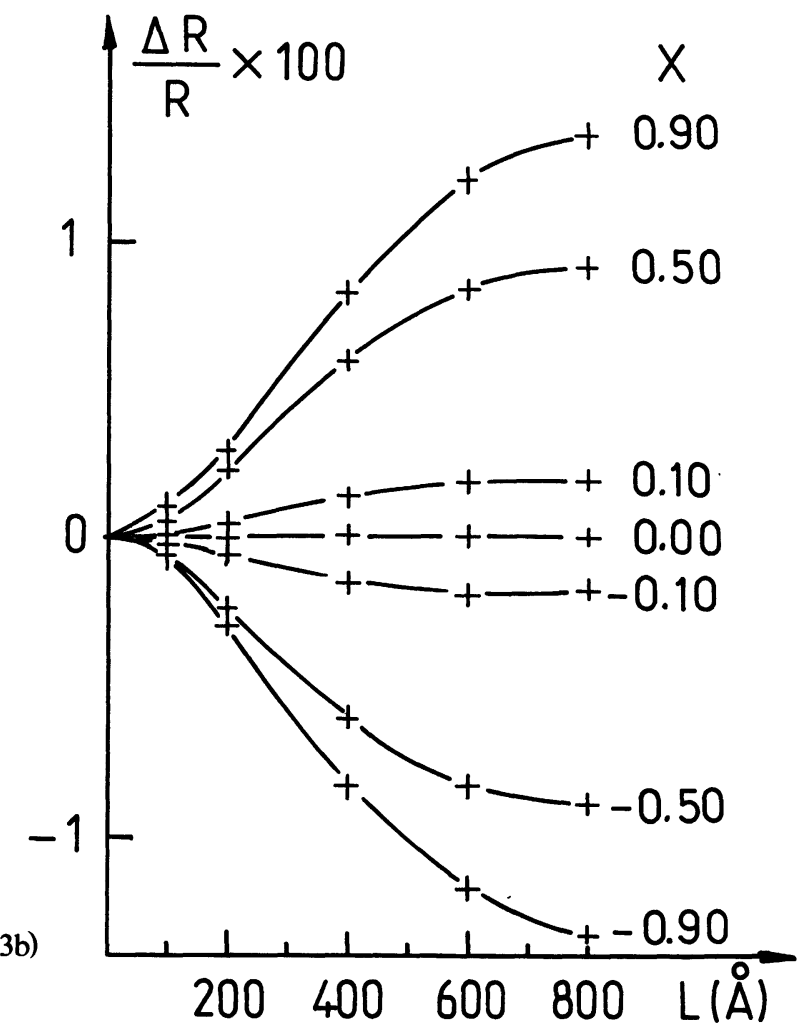

[Relative differences (\%) between the energy reflection coefficient calculated using Webb's approach (normal incidence) and its effective value as a function of the profile thickness $L$, for different dissymmetry parameters $X$ (see Eq. (5)); Fig. 3a $: \Delta n=0.2$; Fig. $3 \mathrm{~b}: \Delta n=0.02$.]

- Pour des épaisseurs comprises entre 100 et $400 \AA$, les courbes relatives à une épaisseur donnée, mais à différentes valeurs du paramètre de dissymétrie, sont très voisines les unes des autres. En ce cas, la réflectométrie permet le calcul d'une épaisseur, sans plus.

- Enfin, pour de fortes épaisseurs $\left(L / \lambda>5 \times 10^{-2}\right)$, la réflectivité dépend à la fois de la nature du profil (ici de son paramètre de symétrie) et de son épaisseur, le profil symétrique ayant, aux faibles incidences, la réflectivité la plus faible. En d'autres termes, la méthode de réflectivité est d'autant plus sélective vis-à-vis du profil interfacial que le rapport $L / \lambda$ est grand. D'où l'intérêt de disposer du paramètre de longueur d'onde, y compris dans le domaine de l'U.V., si une "fenêtre de transparence " est disponible.

La figure 5 présente les variations du rapport $r / r_{F}$ avec l'écart d'indice des milieux extrêmes $\Delta n$, pour différentes épaisseurs de profils symétriques et des angles d'incidence croissants. Le fait remarquable, déjà relevé pour l'incidence normale, est que ce rapport est indépendant de $\Delta n$, dans une large gamme d'épaisseurs et d'angles d'incidence. Ce résultat est d'un grand intérêt car il permet l'évaluation de la réflectivité dans 


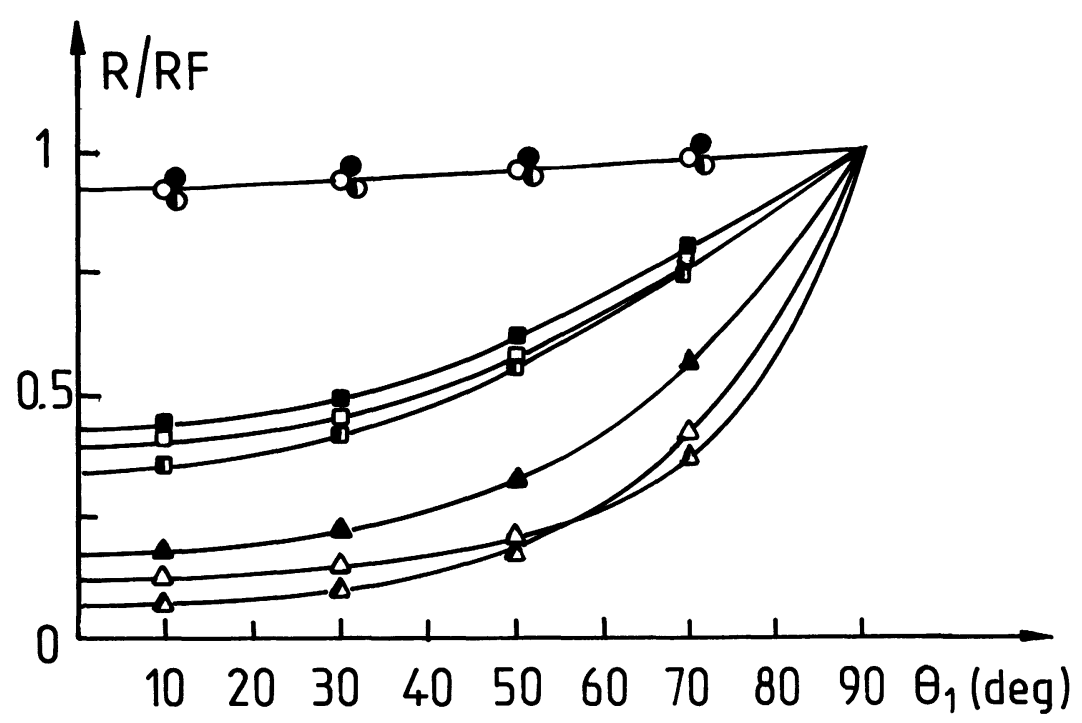

Fig. 4. - Variations du rapport entre le coefficient de réflectivité en énergie relatif au profil sigmoïdal et le coefficient de réflectivité en énergie de Fresnel, pour une onde s, en fonction de l'angle d'incidence, pour différentes valeurs de l'épaisseur $L$ et du paramètre de dissymétrie $X$.
$L=100 \AA: O, X=0,893$;
D, $X=-0,033$;
○, $X=-0,906$
$L=400 \AA: \square, X=0,893$;
口, $X=-0,033$
$\boldsymbol{Q}, X=-0,906$
$L=800 \AA: \triangle, X=0,893$;
$\Delta, X=-0,033$;
$\Delta, X=-0,906$.

[Variations of the ratio between the energy reflection coefficient characteristic of a sigmoidal profile and the related Fresnel reflection coefficient, for an s waver, as a function of the incidence angle, for different thicknesses $L$ and dissymmetry parameters $X$.
$L=100 \AA: O, X=0.893$;
D, $X=-0.033$;
○, $X=-0.906$
$L=400 \AA: \square, X=0.893$;
$\mathbf{\square}, X=-0.033$
घ, $X=-0.906$
$L=800 \AA: \triangle, X=0.893$;
$\Delta, X=-0.003$
$\Delta, X=-0.906$.]

la limite $\Delta n \rightarrow 0$ (approximation de Born) et sa transposition à des situations physiques où $\Delta n$ est beaucoup plus important, par le biais de la relation :

$$
R=\frac{\Delta n}{2 n} \times \lim _{\Delta n \rightarrow 0}\left(\frac{R}{R_{\mathrm{F}}}\right) .
$$

Toutefois, ce résultat n'est pas généralisable à des profils ne présentant pas un centre de symétrie, comme l'illustrent déjà les figures $3 a$ et $3 b$, dans le cas de l'incidence normale [14].

3.2 ONDES DE TYPE « $\mathrm{p}$ ». - Près de l'incidence normale, les réflectivités relatives aux ondes $\mathrm{s}$ et $\mathrm{p}$ sont très voisines, et l'étude des ondes $p$ n'apporte donc pas d'information nouvelle. Par contre, le voisinage de l'incidence brewstérienne constitue, pour les ondes $\mathrm{p}$, un domaine d'investigation particulièrement intéressant, largement exploité en ellipsométrie. La réflectivité de Fresnel $R_{\mathrm{Fp}}$ est nulle à l'incidence brewstérienne (caractérisée par $\operatorname{tg} \theta_{1 \mathrm{~F}}=n_{2} / n_{1}$ ), mais elle passe seulement par un minimum pour une interface diffuse. Si l'on note $R_{\mathrm{p}}^{*}$ et $\theta_{\mathrm{iB}}^{*}$ les valeurs du coefficient de réflectivité en énergie et de l'angle d'incidence relatifs au minimum, il est instructif d'étudier leurs variations avec les caractéristiques du profil sigmoïdal.

La figure 6 illustre les variations du coefficient $R_{\mathrm{p}}^{*}$ avec l'épaisseur de l'interface de profil d'indice symé- trique. Il existe un assez large domaine où $R_{\mathrm{p}}^{*}$ est une fonction quasi affine de $L$. D'autre part, il semble possible de pouvoir mesurer des épaisseurs relativement faibles, inférieures à $100 \AA$, ce qui n'était pas le cas avec une polarisation de type $s$.

La détermination précise du pseudo angle de Brewster $\theta_{1 \mathrm{~B}}^{*}$ permet, par comparaison avec la valeur effective $\theta_{1 \mathrm{~B}}$ de discuter, voire de quantifier, la dissymétrie du profil d'indice. Sur la figure 7, on remarque que la variation de $\theta_{1 \mathrm{~B}}^{*}$ avec $X$ est monotone et que, pour

$$
X=0 . \theta_{1 \mathrm{~B}}^{*} \neq \theta_{1 \mathrm{~B}} .
$$

Donc, une mesure précise de $\theta_{1 \mathrm{~B}}^{*}$ permet d'interpoler une valeur de $X$ en grandeur et en signe. Toutefois, notre calcul a été effectué dans un cas relativement favorable $(L=800 \AA, \Delta n=0,2)$ et il n'est pas certain, par ailleurs, que la précision expérimentale soit suffisante dans les déterminations de $\theta_{1 \mathrm{~B}}$ et $\theta_{1 \mathrm{~B}}^{*}$. Enfin, la figure 6 montre aussi combien les valeurs de $R_{\mathrm{p}}^{*}$ sont fortement dépendantes du paramètre de dissymétrie $X$.

\section{Conclusion.}

Développant actuellement une technique de réflectométrie pour l'étude de la structure d'interfaces polymères, nous voulions en explorer les possibilités. 


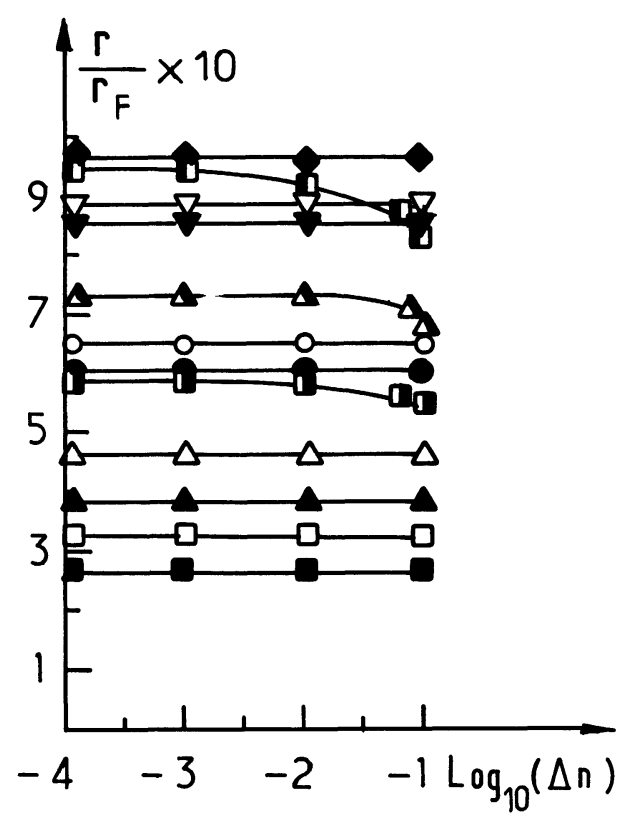

Fig. 5. - Variations du rapport entre le coefficient de réflectivité en amplitude relatif au profil sigmoïdal symétrique et le coefficient de réflectivité en amplitude de Fresnel, pour une onde $s$, en fonction du logarithme décimal de l'écart d'indice, pour différentes épaisseurs et différents angles d'incidence.

$L=100 \AA: \curvearrowright, \theta_{1}=0^{\circ}$

$L=200 \AA: \nabla, \theta_{1}=0^{\circ} ; \nabla, \theta_{1}=30^{\circ}$

$L=400 \AA: 0, \theta_{1}=0^{\circ} ; \mathrm{O}, \theta_{1}=30^{\circ}$

$L=600 \AA: \Delta, \theta_{1}=0^{\circ} ; \triangle, \theta_{1}=30^{\circ}$;

$L=800 \AA: \square, \theta_{1}=0^{\circ} ; \square, \theta_{1}=30^{\circ}$;

$\Delta . \theta_{1}=60^{\circ}$

a, $\theta_{1}=60^{\circ}$

o, $\theta_{1}=80^{\circ}$.

[Variations of the ratio between the amplitude reflection coefficient characteristic of a sigmoidal profile and the corresponding Fresnel reflection coefficient, for an s wave, as a function of the refractive index difference $\Delta n$ (decimal logarithmic scale), for different thickness $L$ and incidence angles $\theta_{1}$.

$L=100 \AA: \vartheta, \theta_{1}=0^{\circ}$

$L=200 \AA: \nabla, \theta_{1}=0^{\circ} ; \nabla, \theta_{1}=30^{\circ}$

$L=400 \AA: 0, \theta_{1}=0^{\circ} ; 0, \theta_{1}=30^{\circ}$

$L=600 \AA: \Delta, \theta_{1}=0^{\circ} ; \Delta, \theta_{1}=30^{\circ} ; \Delta, \theta_{1}=60^{\circ}$;

$L=800 \AA: \square, \theta_{1}=0^{\circ} ; \square, \theta_{1}=30^{\circ} ; \mathbf{D}, \theta_{1}=60^{\circ}$;

$\boldsymbol{\square}, \theta_{1}=80^{\circ}$.]

Fig. 7. - Variations du coefficient de réflectivité en énergie relatif à un profil sigmoïdal, à l'angle pseudo-brewstérien, et de l'angle pseudo-brewstérien $\theta_{1 \mathrm{~B}}^{*}$, avec le paramètre de dissymétrie $X$, pour une épaisseur $L$ de $800 \AA$, et des indices extrêmes égaux à $n_{1}=1,4$ et $n_{2}=1,6$.

[Variations of the energy reflection coefficient characteristic of a sigmoidal profile at the pseudo-brewsterian angle $\theta_{1 \mathrm{~B}}$, and the pseudo-Brewsterian angle $\theta_{1 \mathrm{~B}}$, as a function of the dissymmetry parameter $X$, for $L=800 \AA$ and asymptotic refractive indexes of $n_{1}=1.4$ and $n_{2}=1.6$.]

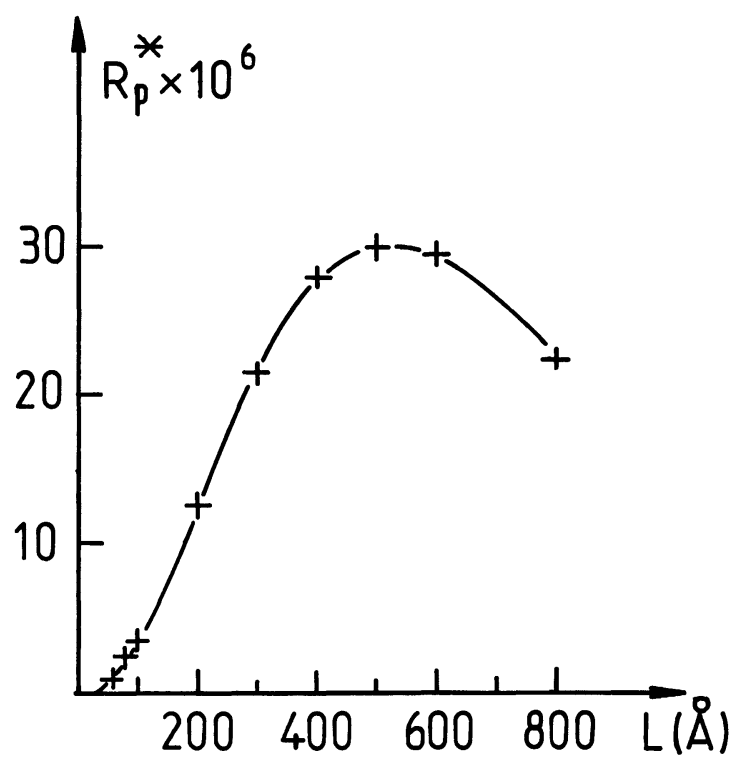

Fig. 6. - Variations du coefficient de réflectivité en énergie relatif au profil sigmoïdal symétrique, pour une onde $p$, à l'angle d'incidence pseudo-brewstérienne, en fonction de l'épaisseur du profil.

[Variations of the energy reflection coefficient characteristic of a sigmoidal profile, for a $\mathrm{p}$ wave at the pseudo-brewsterian angle $\theta_{1 \mathrm{~B}}$, as a function of the profile thickness $L$.]

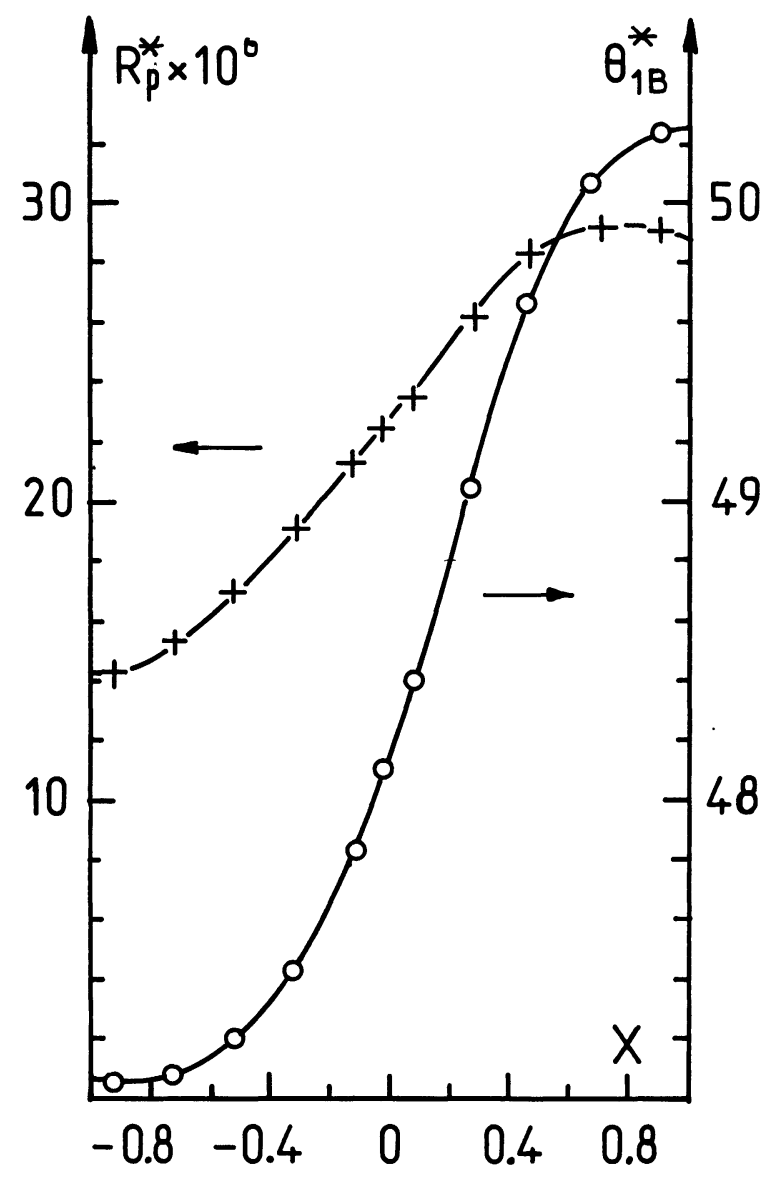


Plusieurs résultats émergent de notre analyse :

- Pour le calcul du coefficient de réflectivité d'une interface caractérisée par son profil d'indice de réfraction, la méthode d'Abelès dite des cosinus est la plus générale, donc la plus fiable.

- Dans le cas de profils interfaciaux symétriques, dont la structure est définie par une seule longueur caractéristique, le coefficient de réflectivité est directement proportionnel à la transformée de Fourier de la fonction $(\mathrm{d} n / \mathrm{d} z)$, dérivée du profil d'indice. Une seule mesure de coefficient de réflectivité suffit alors, en principe, pour déterminer l'épaisseur du profil, lorsqu'on connaît la forme analytique de la fonction $n(z)$.

- Avec des structures interfaciales plus complexes, la proportionnalité entre $R_{i}(i=\mathrm{s}, \mathrm{p})$ et la transformée de Fourier de $(\mathrm{d} n / \mathrm{d} z)$ n'est valable que dans la limite $(\Delta n / n) \rightarrow 0$. En particulier, nous avons montré que pour un profil de type sigmoïdal faisant intervenir deux longueurs caractéristiques, le coefficient de réflectivité dépend fortement de l'épaisseur $L$ et du paramètre de dissymétrie $X$.

Nous n'avons pas fait mention, jusqu'alors, de la contribution qu'apportent les ondes thermiques capillaires à la réflectivité d'une interface fluide/fluide. Différentes approches théoriques [22,23] et quelques contributions expérimentales [24] existent cependant sur ce sujet encore controversé. En se plaçant dans le cadre de l'approche de Webb, Meunier [22] a étudié la contribution des ondes capillaires à la réflectivité d'interfaces diffuses. Il a montré que le calcul du coefficient de réflectivité se ramène à celui d'une interface diffuse dont les caractéristiques structurales doivent être redéfinies, tout en restant proches de celles considérées dans cet article. Si l'on applique un résultat établi par Buff, Lovett et Stillinger [25] au cas de l'interface résultant de la séparation de phase d'une solution polymère en mauvais solvant, il apparait que la contribution des ondes de surface à l'épaisseur interfaciale est dominante. Il s'agit donc là d'un thème d'investigation encore largement ouvert.

\section{Appendice I}

Les matrices $M_{j \mathrm{~s}}$ et $M_{j \mathrm{p}}$ ont été calculées par Abelès [18]. Ces matrices ont pour expression :

$$
\begin{aligned}
& M_{j \mathrm{~s}}=\left[\begin{array}{cc}
\cos \left(f_{j} t_{j}\right) & +i g_{j}^{-1} \sin \left(f_{j} t_{j}\right) \\
+i g_{j} \sin \left(f_{j} t_{j}\right) & \cos \left(f_{j} t_{j}\right)
\end{array}\right] \\
& M_{j \mathrm{p}}=\left[\begin{array}{cc}
\cos \left(f_{j} t_{j}\right) & i h_{j}^{-1} n_{j}^{2} \sin \left(f_{j} t_{j}\right) \\
i h_{j} n_{j}^{-2} \sin \left(f_{j} t_{j}\right) & \cos \left(f_{j} t_{j}\right)
\end{array}\right]
\end{aligned}
$$

avec $t_{j}$ l'épaisseur de la tranche homogène $j$

$$
\begin{gathered}
f_{j}=\frac{2 \pi}{\lambda}\left(n_{j}^{2}-n_{1}^{2} \sin ^{2} \theta_{1}\right)^{1 / 2}=k_{0}\left(n_{j}^{2}-n_{1}^{2} \sin ^{2} \theta_{1}\right)^{1 / 2} \\
g_{j}=\left(n_{j}^{2}-n_{1}^{2} \sin ^{2} \theta_{1}\right)^{1 / 2}\left(\frac{\varepsilon_{0}}{\mu_{0}}\right)^{1 / 2}
\end{gathered}
$$

REVUE DE PHYSIQUE APPLIQUÉE. - T. 20, № 9, SEPTEMBRE 1985

$$
h_{j}=\left(n_{j}^{2}-n_{1}^{2} \sin ^{2} \theta_{1}\right)^{1 / 2}\left(\frac{\mu_{0}}{\varepsilon_{0}}\right)^{1 / 2}
$$

$n_{1}$ étant l'indice du milieu incident et $\theta_{1}$ l'angle d'incidence.

\section{Appendice II.}

Etablissons les formules proposées par Webb [6] pour des ondes polarisées perpendiculairement au plan d'incidence, formules utilisées par plusieurs auteurs $[1,21]$.

En partant des équations de Maxwell, Brekhovskikh [10] aboutit à une équation différentielle dont l'inconnue est le coefficient de réflectivité complexe $V(z)$ (voir Fig. 1) :

$$
V(z)=\frac{E_{x}^{\mathrm{i}}(z)}{E_{x}^{\mathrm{r}}(z)} .
$$

Cette équation s'écrit :

$$
\frac{\mathrm{d} V}{\mathrm{~d} z}=-2 i \beta V+j\left(1-V^{2}\right)
$$

avec

$$
\beta=k_{0} n(z) \cos \theta(z) \quad \text { et } \quad j=\frac{1}{2} \frac{d \ln \beta}{\mathrm{d} z} .
$$

Cette équation peut encore s'écrire :

$$
\begin{aligned}
& V(z) \exp \left(2 \int_{z_{0}}^{z} \beta \mathrm{d} z\right)= \\
& \quad=\int_{z_{0}}^{z} j\left(1-V^{2}\right) \exp \left(2 i \int_{z_{0}}^{z} \beta \mathrm{d} z\right) \mathrm{d} z .
\end{aligned}
$$

On résout cette équation par approximations successives, l'ordre 0 correspondant à $V=0$.

Quand on se restreint à l'ordre 1 on obtient

$$
V(z)=\exp (-i s(z)) \int_{z_{0}}^{z} \gamma(z) \exp (i s(z)) d z
$$

d'où

$$
|R|^{2}=\left|\int_{z_{0}}^{z} \gamma(z) \exp i s(z) \mathrm{d} z\right|^{2}
$$

avec

$$
s(z)=2 \int_{z_{0}}^{z} \beta \mathrm{d} z
$$

En posant $n(z)=n_{0}+\Delta n f(z)$ et en supposant $\Delta n \ll 1$, l'équation (II.5) peut s'écrire :

$$
|R|^{2}=\left|\frac{\Delta n}{2 n_{0} \cos \theta_{0}} \int_{z_{0}}^{z} f^{\prime}(z) \exp \left(2 i k_{0} \cos \theta_{0} z\right) \mathrm{d} z\right|^{2} \text {. }
$$

Cette formule correspond à la formule de Webb dans le cas où $\Delta n \ll 1$, c'est-à-dire dans le cas où $k_{0} \simeq k$. 


\section{Bibliographie}

[1] Meunier, J. Colloides et Interfaces (Les éditions de physique, Les Ulis) 1984, p. 181.

[2] Bouchiat, M. A., Langevin, D., J. Colloid Int. Sci. 63 (1978) 193

[3] Beaglehole, D., J. Physique Colloque 44 (1983) C-10.

[4] LeE, J., Fuller, G. G., Macromolecules 17 (1984) 375.

[5] DéJardin, P., Thèse Université Louis Pasteur, Strasbourg (1981).

[6] Huang, J. S., Webb, W. W., J. Chem. Phys. 50 (1969) 3677.

[7] Wu, E. S., Webr, W. W., J. Physique Colloque 33 (1972) 149

[8] Beaglehole, D., J. Phys. Chem. 87 (1983) 4749.

[9] Azzam, R. M. A., Bashara, N. H., Ellipsometry and polarized light (North-Holland) 1977.

[10] BReKhovskikH, L. H., Waves in layered media (Academic Press, London) 1960.

[11] De Gennes, P. G., Physics Today 36 (1983) 33.

[12] SPIEGLER, K. S., Study of Membrane-Solution Interfaces by electrochemical methods. United States Department of the Interior, office of saline water research and development, Report No 353, Superintendent of documents, Washington D.C. (1968).

[13] Fermi, E., Notes on Quantum Mechanics (University of Chicago Press, Chicago) 1961.

[14] Partant d'une théorie de champ moyen (approche de Flory) nous avons adopté l'analyse développée par Joanny et Leibler [17] à la séparation de phase loin du point critique. Les calculs montrent que les longueurs de corrélation peuvent alors être nettement différentes dans l'une et l'autre phase.

[15] DéJardin, P., SchaAf, P., résultats non publiés.

[16] Il est possible d'utiliser comme fonction de distribution $\left|n_{i}-n_{i}(z)\right|$ dans chacun des milieux extrêmes $(i=1,2)$. L'expression du moment d'ordre $m$ s'écrit alors

$\left\langle z^{m}\right\rangle=\int_{-\infty}^{0}\left[n(z)-n_{1}\right] z^{m} \mathrm{~d} z+\int_{0}^{+\infty}\left[n_{2}-n(z)\right] z^{m} \mathrm{~d} z$.

[17] Joanny, J. F., Leibler, L., J. Physique 39 (1978) 951.

[18] Abelès, F., Ann. Phys. 5 (1950) 596.

[19] Drude, P., Wied. Ann. 43 (1891) 126.

[20] Charmet, J. C., De Gennes, P. G., J. Opt. Soc. Am. 73 (1983) 1777.

[21] Schmitr, A. Colloides et interfaces (Les éditions de physique, Les Ulis) 1984, p. 181.

[22] Meunier, J., C.R. Hebd. Séan. Acad. Sci. Paris 292 (1981) 1469.

[23] Jasnow, D., Rudnick, J., Phys. Rev. Lett. 41 (1978) 698.

[24] Beaglehole, D., Physica 112B (1982) 320.

[25] Buff, F. P., Lovett, R. A., Stillinger, F. H., Phys. Rev. Lett. 15 (1965) 621. 